the same disease was obtained in 2 cases $(50 \%$ of NF1 and $100 \%$ of IP).

Conclusions The leading NCS in this study was SWS.70\% of children with NCS presented with seizures. The commonest type of seizure among them is GTCS. Children with TSC had seizures refractory to anticonvulsants. Careful evaluation of NCS children can reveal problems like Learning disabilities.

Recommendation All children with neurocutaneous markers should be evaluated and investigated in detail to detect neurological affection.

Keywords Neurocutaneous syndromes, Hypomelanosis of Ito, Sturge Weber Syndrome, Tuberous Sclerosis Complex.

\section{P0-0819 CASE SERIES OF CHILDREN WITH NEUROCUTANEOUS SYNDROMES}

N Divakar. Paediatrics, Pushpagiri Institute of Medical Sciences, Cochin, India

10.1136/archdischild-2014-307384.1453

Introduction Neurocutaneous syndromes (NCS) are a heterogenous group of disorders characterised by abnormalities of both the integument and central nervous system that are believed to originate from a defect in differentiation of the primitive ectoderm. Cutaneous manifestations usually appear early in life and progress with time, but neurological features generally present at a later age.

Aim To study the clinical profile of children with neurocutaneous syndromes and their various symptomatology, the seizure types and the response to treatment.

Subjects and methods A retrospective crosssectional study was conducted in the Department of Paediatrics, Pushpagiri Medical College Hospital, Tiruvalla, during the period from January 2013 to June 2013. Children between the age group 0 and 15 years were included in the study on the basis of standard diagnostic criteria for different NCS. Investigations done were CT, MRI, EEG,and skin biopsy for appropriate cases

Results The study population comprised of 10 children (5 boys, 5 girls). The various forms of NCS observed were Sturge Weber syndrome (SWS) - 4 Neurofibromatosis (NF1)- 2, Hypomelanosis of Ito (HOI) -2 , Tuberous sclerosis complex (TSC) -1 , and Incontinentia pigmenti (IP) -1 . A total of 8 children (80\%) presented with neurological symptoms and the remaining $2(20 \%)$ presented with cutaneous symptoms of which 1 was found to have learning disability on evaluation. The neurological problems were, $70 \%$ had seizures of which $100 \%$ were SWS and TSC, 50\% were HOI and NF1. 72\% had generalised tonic clonic seizures (GTCS) and 28\% had focal seizures. The child with TSC showed refractory epilepsy. Developmental delay was detected in 50\% of cases and maximum delay was seen in HOI. Family history of the same disease was obtained in 2 cases $(50 \%$ of NF1 and $100 \%$ of IP).

Conclusions The leading NCS in this study was SWS.70\% of children with NCS presented with seizures. The commonest type of seizure among them is GTCS. Children with TSC had seizures refractory to anticonvulsants. Careful evaluation of NCS children can reveal problems like Learning disabilities.

Recommendation All children with neurocutaneous markers should be evaluated and investigated in detail to detect neurological affection.

Keywords Neurocutaneous syndromes, Hypomelanosis of Ito, Sturge Weber Syndrome, Tuberous Sclerosis Complex.
P0-0820 SLEEP COMPLAINTS AMONG THE NEUROLOGICALLY IMPAIRED CHILDREN: QUESTIONNAIRE-BASED STUDY

${ }^{1} \mathrm{~T}$ Ediberidze, ${ }^{1} \mathrm{~N}$ Gogatishvili, ${ }^{2} \mathrm{~N}$ Tatishvili, ${ }^{3} \mathrm{~L}$ Maisuradze, ${ }^{1} \mathrm{~S}$ Kasradze. ${ }^{1}$ Epilepsy Centre, Institute of Neurology and Neuropsychology, Tbilisi, Georgia; '2Department of Neuroscience, M. Iashvili Children Central Hospital, Tbilisi, Georgia; ${ }^{3}$ llia State University, Tbilisi, Georgia

10.1136/archdischild-2014-307384.1454

Background and aims Many studies have shown a significant increase in SD in neurologically impaired patients. The aim of study was to evaluate the prevalence of sleep disturbances (SD) among the children with cerebral palsy (CP) with or without comorbidity with Epilepsy.

Methods A structured sleep-waking questionnaire was developed on the basis of Child Sleep Questionnaire for Parents and the Paediatric Sleep Questionnaire. Subjects were recruited from the Institute of Neurology and Neuropsychology/Tbilisi/Georgia.

Results 165 1-to-8-years-old children with CP and 359 age and sex-matched subjects were selected. 85 patients with CP had Epilepsy. Different SD were identified in $64.8 \%(\mathrm{n}=107)$ of children with CP vs. $20.3 \%(n=73)$ in healthy children. Sleep disorder breathing (SDB) was the commonest among the children with CP $58.78 \%(\mathrm{n}=97)$ vs. $8.07 \%(\mathrm{n}=29)$ in controls. Other main problems in neurologically impaired children were problems with sleep onset 41.2\% $(n=68)$ and sleep maintenance $39.4 \%(n=65)$. The frequency of both was about 4 -fold higher than in healthy controls. Sleep onset difficulty was commonest among controls $11.4 \%(n=41)$.

SDB was more prevalent among the children with $\mathrm{CP}$ and Epilepsy $69.4 \%(\mathrm{n}=59)$ vs. $47.5 \%(\mathrm{n}=38)$ in children without seizures. Problems with sleep onset and maintenance were a little higher among the subjects without Epilepsy 48.75\% ( $\mathrm{n}=$ $39)$ and $42.5 \%(n=34)$ correspondingly vs. $34.12 \%(n=29)$ and $36.47 \%(\mathrm{n}=31)$ in children with CP and Epilepsy.

Conclusions Study shows that SD is more prevalent in neurologically impaired children. Children with CP and Epilepsy show to be at higher risk to have SDB, compared to children with $\mathrm{CP}$ alone;

\section{P0-0821 THE APPROACH TO THE CHILD WITH EPILEPSY (APCWEP) AUDIT IN A BUSY UAE GENERAL HOSPITAL}

T El-Azzabi, V Malpani, MS Kasim, R Ravindran. Child Health Institute, Alain Hospital, AlAin, United Arab Emirates

\subsection{6/archdischild-2014-307384.1455}

Background and aims Epilepsy is a common neurological disorder. We are developing services for children with epilepsy (CWE) at Al-Ain hospital. Aiming to improve services we conducted an audit to evaluate the assessment of all CWE against NICE guidelines.

Methods Cases were identified from the hospital database. Retrospectively, electronic case notes of children presented with a diagnosis of seizure (s) between 1/7/2011 and 31/3/2014 were reviewed. Each note of all children meeting inclusion criteria was evaluated, twice independently, using modified Epilepsy 12 performance indicators.

Results 395 children were identified with a diagnosis of seizure(s). 91 fulfilled entry criteria.

1. 70/91 were referred to epilepsy-expert paediatricians.

2. 58/70 were seen within 2 weeks.

3. None seen by epilepsy nurse (Unavailable). 